\title{
Author Correction: Ultrafast hole spin qubit with gate-tunable spin-orbit switch functionality
}

\author{
Florian N. M. Froning, Leon C. Camenzind, Orson A. H. van der Molen (D), Ang Li, Erik P. A. M. Bakkers (D), \\ Dominik M. Zumbühl (1D and Floris R. Braakman (D)
}

Correction to: Nature Nanotechnology https://doi.org/10.1038/s41565-020-00828-6, published online 11 January 2021.

In the version of this Article originally published, the dependence of the Rabi frequency of the hole spin qubit on the $g$-factor was stated incorrectly. We wrote that the Rabi frequency is proportional to the $g$-factor perpendicular to the external magnetic field $\left(g_{\perp}\right)$, but, in fact, it is the $g$-factor parallel to the external magnetic field $\left(g_{\|}\right)$as shown in ref. 25 (Golovach, V. N, Borhani, M. \& Loss, D.). Thus, the following sentence has been amended from "This effective field $\mathbf{B}_{\text {eff }}$ drives the Rabi oscillations, with Rabi frequency $f_{\text {Rabi }}=g_{\perp} \mu_{\mathrm{B}} B_{\text {eff }}(t) / 2 h$, with $g_{\perp}$ the $g$-factor along the direction of $\mathbf{B}_{\text {eff }}$ and thus perpendicular to $\mathbf{B}_{\text {ext }}$, and $h$ Planck's constant." to "This effective field $\mathbf{B}_{\text {eff }}$ drives the Rabi oscillations, with Rabi frequency $f_{\text {Rabi }}=g_{\|} \mu_{\mathrm{B}} B_{\text {eff }}(t) / 2 h$, with $g_{\|}$the $g$-factor along the direction of $\mathbf{B}_{\text {ext, }}$ and $h$ Planck's constant." Accordingly, the phrase "the $g$-factor along $\mathbf{B}_{\text {ext }}$ " has been deleted from the sentence starting "Here, $f_{\mathrm{MW}}$ and $P_{\mathrm{MW}}$ are kept fixed...".

When measuring at a constant value of the external magnetic field $B_{\text {ext }}, f_{\text {Rabi }}$ and $l_{\text {so }}$ still depend on the $g$-factor (as in the original version). However, when measuring at a fixed resonant microwave frequency $f_{\mathrm{MW}}=g_{\|} \mu_{\mathrm{B}} B_{\mathrm{ext}} / h$, any change of the $g$-factor is compensated for by an inversely proportional change of $B_{\text {ext }}$, and the apparent $g$-factor dependence vanishes from the equation of the Rabi frequency, thus making the observation of a gate-voltage-dependent spin-orbit coupling strength more robust. This is now reflected in the corrected Article, as described below.

The following changes have been made: (1) in the sentence beginning "We extracted a spin-orbit length..., ' $3 \mathrm{~nm}$ ' has been changed to ' $4 \mathrm{~nm}$ '; (2) in the sentence beginning "For SOI-mediated spin rotations", ' $g_{\perp}$ ' has been changed to ' $g_{\|}$'; ( 3 ) in the sentence beginning "We found remarkably short..., ' $28 \mathrm{~nm}$ ' and ' $3 \mathrm{~nm}$ ' have been changed to ' $23 \mathrm{~nm}$ ' and ' $4 \mathrm{~nm}$ ', respectively. Also, the following paragraph has been amended from

"Therefore, the Rabi frequency depends on $l_{\mathrm{so}}$, the a.c. electric field amplitude $E_{\mathrm{MW}}(t)$ created through the periodic gate voltage modulation, $B_{\text {ext, }}$, the quantum dot confinement $\Delta_{\text {orb }}$ and the $g$-factor. Despite an observed change of $g_{\|}$with $V_{\mathrm{M}}$ (refs. ${ }^{18,43}$ ) by a factor of 1.5 (Fig. 3c), the effect on the Rabi frequency was small: at a constant Zeeman energy, we can write $f_{\text {Rabi }} \propto f_{\text {MW }} g_{\perp} / g_{\|}$. Hence, if the $g$-factor anisotropy $g_{\perp} / g_{\|}$is only weakly affected by gate voltages, as observed here (Supplementary Fig. 3d,e), then the Rabi frequency change is correspondingly small."

to

"For measurements at a fixed microwave frequency $f_{\mathrm{MW}}$, as done here, the apparent $g$-factor dependence vanishes and the Rabi frequency depends only on the spin-orbit length $l_{\mathrm{so}}$, the quantum dot orbital level splitting $\Delta_{\mathrm{orb}}$ and the a.c. electric field $E_{\mathrm{MW}}(t) \mathrm{created}$ through the periodic gate voltage modulation (see Supplementary equation (1)."

Subsequent to the above change, reference 43 now becomes reference 45 , and has now been cited as follows: "Moreover, the variation of $g_{\|}$(ref. 45) in Fig. 3c..." References 44 and 45 have been renumbered to 43 and 44, respectively.

Also, in the Supplementary Information, the correction to the $g$-factor results in its elimination from Supplementary equation (1), making the Rabi frequency independent of the $g$-factor for our measurements where the microwave frequency was held fixed, as previously discussed; the corrected equation is:

$$
f_{\text {Rabi }}=f_{\mathrm{MW}} \cdot \frac{l_{\mathrm{dot}}}{l_{\mathrm{so}}} \cdot \frac{e\left|\mathbf{E}_{\mathrm{MW}}(t)\right| l_{\mathrm{dot}}}{\Delta_{\mathrm{orb}}}
$$

The paragraph following Supplementary equation (1) has been updated to read "Here, we used $f_{\text {Rabi }}=g_{||} \mu_{\mathrm{B}}\left|\mathbf{B}_{\text {eff }}(t)\right| /(2 h)$ with $\left|\mathbf{B}_{\text {eff }}(t)\right|$ as defined in Eq. 1 of the main text, and $\left|\mathbf{B}_{\text {ext }}(t)\right|$ is converted to $f_{\mathrm{MW}}$ via the resonance condition $g_{||} \mu_{\mathrm{B}}\left|\mathbf{B}_{\text {ext }}(t)\right|=h f_{\mathrm{MW}}$, since $f_{\mathrm{MW}}$ is held fixed. In order to stay on resonance, we compensate the variation of $g_{\|}$(shown in Figure $3 \mathrm{c}$ of the main text) with a proportional change of $\left|\mathbf{B}_{\text {ext }}(t)\right|$ in the opposite direction, effectively making $f_{\text {Rabi }}$ independent of $g_{\|}$. We note that $l_{\text {dot }}=\hbar / \sqrt{\Delta_{\text {orb }} m_{\text {eff }}}$ with the effective hole mass $m_{\text {eff." }}$

The related discussion in the Supplementary Information has also been updated as follows: (1) the paragraph beginning "Lastly, the Rabi frequency..." has been changed to "In conclusion, we estimate the contributions from changes in electric feld amplitude (no change) and orbital level splitting to account for a total of not more than a 1.5-fold change of the Rabi frequency. The very large change of the Rabi frequency by a factor of 7 can therefore be attributed mainly to an electrically tunable spin-orbit interaction, as expected from a direct Rashba type of spin-orbit interaction"; (2) the sentence beginning "In order to see the effect of..." has been changed to "We also study the evolution of the $g$-factor in other directions as a function of $V_{M^{*}}$ "; (3) the sentence beginning "Figure S3 c shows these..." has been changed to "Figure S3 c shows $g$-factors in two directions that are perpendicular to the orientation of $\mathbf{B}_{\text {ext }}$ as function of $V_{\mathrm{M}}$, extracted from measurements of the Larmor frequency."; (4) the sentences beginning "These two directions..." and "While these changes in anisotropy..." 
have been deleted; (5) in the sentence beginning “The corresponding values..., " $|\mathrm{E}(t)|, g, \Delta_{\text {orb }}$ " has been changed to " $|\mathrm{E}(t)|, \Delta_{\text {orb }}$ "; $(6)$ the sentence beginning "For the $g$-factor..." has been changed to "For $\Delta_{\text {orb }}$ we take the values shown in Figure S3 b, which were obtained from measurements of bias triangles"; (6) in the sentence beginning "For the measurement with $V_{\mathrm{M}}$..." ' $3 \mathrm{~nm}$ (20 nm)' has been changed to ' 4 $\mathrm{nm}(26 \mathrm{~nm})$; (7) in the Figure S4 caption, "measured values of $g, \Delta_{\text {orb }}$ " has been changed to "measured values of $\Delta_{\text {orb }}$ ".

The conclusions of the Article are unaffected.

The online versions of the Article have been amended and the Supplementary Information file replaced.

Published online: 5 July 2021

https://doi.org/10.1038/s41565-021-00930-3

(c) The Author(s), under exclusive licence to Springer Nature Limited 2021

\section{Author Correction: The current landscape of nucleic acid therapeutics}

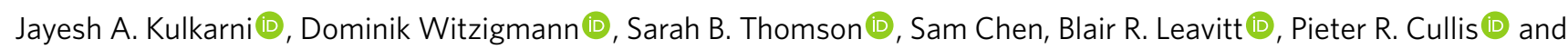
Roy van der Meel(D)

Correction to: Nature Nanotechnology https://doi.org/10.1038/s41565-021-00898-0, published online 31 May 2021.

In Table 2 of the version of this Review originally published, the nucleic acid therapeutic 'Nedosiran' was incorrectly listed as a GalNAcASO conjugate, but it is a GalNAc-siRNA conjugate. Table 2 has now been amended accordingly in the online versions of the Review.

Published online: 30 June 2021

https://doi.org/10.1038/s41565-021-00937-w

(c) The Author(s), under exclusive licence to Springer Nature Limited 2021 